\title{
Some Basic Properties of Certain New Subclass of Meromorphic Functions
}

\author{
Lei Shi ${ }^{1}$ and Zhi-Gang Wang ${ }^{2}$ \\ ${ }^{1}$ School of Mathematics and Statistics, Anyang Normal University, Anyang, Henan 455000, China \\ ${ }^{2}$ School of Mathematics and Computing Science, Hunan First Normal University, Changsha, Hunan 410205, China \\ Correspondence should be addressed to Lei Shi; shimath@163.com
}

Received 13 December 2014; Accepted 11 February 2015

Academic Editor: Giuseppe Marino

Copyright (c) 2015 L. Shi and Z.-G. Wang. This is an open access article distributed under the Creative Commons Attribution License, which permits unrestricted use, distribution, and reproduction in any medium, provided the original work is properly cited.

We introduce and investigate a new subclass $\mathscr{M}(\beta, \eta)$ of meromorphic functions. Some interesting properties such as inclusion relationship, coefficient estimates, neighborhoods, and partial sums are proved. Connections of the results with known results are also considered.

\section{Introduction}

Let $\Sigma$ denote the class of functions $f$ of the form

$$
f(z)=\frac{1}{z}+\sum_{k=1}^{\infty} a_{k} z^{k}
$$

which are analytic in the punctured open unit disk:

$$
\mathbb{U}^{*}:=\{z: z \in \mathbb{C}, 0<|z|<1\}=: \mathbb{U} \backslash\{0\} .
$$

Let $\mathscr{P}$ denote the class of functions of the form

$$
p(z)=1+\sum_{k=1}^{\infty} p_{k} z^{k}
$$

which are analytic $\mathbb{U}$ and satisfy the condition

$$
\mathfrak{R}(p(z))>0 \quad(z \in \mathbb{U}) .
$$

A function $f \in \Sigma$ is said to be in the class $\mathscr{M} \mathcal{S}^{*}(\alpha)$ of meromorphic starlike functions of order $\alpha$ if it satisfies the inequality

$$
\mathfrak{R}\left(\frac{z f^{\prime}(z)}{f(z)}\right)<-\alpha \quad\left(z \in \mathbb{U}^{*} ; 0 \leq \alpha<1\right) .
$$

For $\eta>1$, Wang et al. [1] (see also Nehari and Netanyahu [2]) introduced and studied a new subclass $\mathscr{M}(\eta)$ of $\Sigma$ consisting of functions $f$ satisfying

$$
\mathfrak{R}\left(\frac{z f^{\prime}(z)}{f(z)}\right)>-\eta \quad\left(z \in \mathbb{U}^{*}\right) .
$$

We note that meromorphic starlike functions and related topics attract many authors' attentions; see (for example) the earlier works [3-8] and the references cited therein.

Let

$$
\mathfrak{f}(z)=z+\sum_{k=m+1}^{\infty} a_{k} z^{k} \quad(m \in \mathbb{N}:=\{1,2,3 \ldots\})
$$

be analytic in $\mathbb{U}$. Assuming that $\alpha \in \mathbb{C}$ and $0 \leq \beta<1$, we say that a function $f \in \mathscr{H}_{m}(\alpha, \beta)$ if it satisfies the condition

$$
\begin{array}{r}
\Re\left(\frac{z f^{\prime}(z)}{f(z)}+\alpha \frac{z f^{\prime \prime}(z)}{f(z)}\right)>\alpha \beta\left(\beta+\frac{m}{2}-1\right)+\beta-\frac{m}{2} \\
(z \in \mathbb{U}) .
\end{array}
$$

The function class $\mathscr{H}_{m}(\alpha, \beta)$ was introduced and studied recently by Ravichandran et al. [9], Liu et al. [10], Singh and Gupta [11], and Wang et al. [12]. 
In [13], Wang et al. introduced a subclass of meromorphic function $\widetilde{\mathscr{H}}(\beta, \lambda)$ which satisfies the condition

$$
\begin{array}{r}
\mathfrak{R}\left(\frac{z f^{\prime}(z)}{f(z)}+\beta \frac{z^{2} f^{\prime \prime}(z)}{f(z)}\right)<\beta \lambda\left(\lambda+\frac{1}{2}\right)+\frac{\beta}{2}-\lambda \\
\left(\beta \geq 0 ; \frac{1}{2} \leq \lambda<1 ; z \in \mathbb{U}^{*}\right) .
\end{array}
$$

It was proved that the class $\widetilde{\mathscr{H}}(\beta, \lambda)$ is a subclass of the $\mathscr{M} \mathcal{S}^{*}(\lambda)$ of meromorphically starlike functions of order $\lambda$.

Motivated essentially by the above works, we introduce and investigate a new subclass of $\Sigma$ of meromorphic functions.

Definition 1. Suppose that $\eta>1$. Let $\mathscr{M}(\beta, \eta)$ denote a subclass of $\Sigma$ consisting of functions satisfying the condition that

$$
\begin{array}{r}
\mathfrak{R}\left(\frac{z f^{\prime}(z)}{f(z)}+\beta \frac{z^{2} f^{\prime \prime}(z)}{f(z)}\right)>\frac{1}{2} \beta\left(2 \eta^{2}+\eta+1\right)-\eta \\
\left(z \in \mathbb{U}^{*}\right) .
\end{array}
$$

We note that, for $\beta=0$, the class $\mathscr{M}(0, \eta)$ reduces to $\mathscr{M}(\eta)$.

In the present paper, we aim at proving some interesting properties such as inclusion relationship, coefficient estimates, neighborhoods, and partial sums for functions in the class $\mathscr{M}(\beta, \eta)$. tion.

The following lemmas will be required in our investiga-

Lemma 2 (see [14]). Let $\Omega$ be a set in the complex plane $\mathbb{C}$ and suppose that $\Phi$ is mapping from $\mathbb{C}^{2} \times \mathbb{U}$ to $\mathbb{C}$ which satisfies $\Phi(i x, y ; z) \notin \Omega$ for $z \in \mathbb{U}$ and for all real $x, y$ such that $y \leq$ $-\left(1+x^{2}\right) / 2$. If the function $\psi(z)=1+c_{1} z+c_{2} z^{2}+\cdots$ is analytic in $\mathbb{U}$ and $\Phi\left(\psi(z), z \psi^{\prime}(z) ; z\right) \in \Omega$ for all $z \in \mathbb{U}$, then $\mathfrak{R}(\psi(z))>0$.

Lemma 3. Let $\eta>1,0 \leq \beta<1$, and $-1+2 \beta+\gamma>0$. Suppose also that the sequence $\left\{A_{k}\right\}_{k=1}^{\infty}$ is defined by

$$
\begin{gathered}
A_{1}=\frac{-1+2 \beta+\gamma}{1-\beta}, \\
A_{k}=\frac{2(-1+2 \beta+\gamma)}{(k+1)[1+(k-2) \beta]}\left[1+\sum_{m=1}^{k-1} A_{m}\right] .
\end{gathered}
$$

Then

$$
\begin{aligned}
A_{k}= & \frac{-1+2 \beta+\gamma}{1-\beta} \\
& \cdot \prod_{m=1}^{k-1} \frac{(m+1)[1+(m-2) \beta]-2(1-2 \beta-\gamma)}{(m+2)[1+(m-1) \beta]}
\end{aligned}
$$

$(k \geq 2)$.
Proof. From (11), we have

$$
\begin{aligned}
& (k+1)[1+(k-2) \beta] A_{k}=2(-1+2 \beta+\gamma)\left[1+\sum_{m=1}^{k-1} A_{m}\right], \\
& (k+2)[1+(k-1) \beta] A_{k+1}=2(-1+2 \beta+\gamma)\left[1+\sum_{m=1}^{k} A_{m}\right] .
\end{aligned}
$$

Combining (13), we find that

$$
\frac{A_{k+1}}{A_{k}}=\frac{(k+1)[1+(k-2) \beta]+2(-1+2 \beta+\gamma)}{(k+2)[1+(k-1) \beta]} \quad(k \in \mathbb{N}) .
$$

Thus, for $k \geq 2$, we deduce from (14) that

$$
\begin{aligned}
A_{k}= & \frac{A_{k}}{A_{k-1}} \times \frac{A_{k-1}}{A_{k-2}} \times \cdots \times \frac{A_{2}}{A_{1}} \times A_{1} \\
= & \frac{k[1+(k-3) \beta]+2(-1+2 \beta+\gamma)}{(k+1)[1+(k-2) \beta]} \\
& \times \frac{(k-1)[1+(k-4) \beta]+2(-1+2 \beta+\gamma)}{k[1+(k-3) \beta]} \\
& \times \cdots \times \frac{2(1-\beta)+2(-1+2 \beta+\gamma)}{3[1+0 \beta]} \times \frac{-1+2 \beta+\gamma}{1-\beta} \\
= & \frac{-1+2 \beta+\gamma}{1-\beta} \\
& \cdot \prod_{m=1}^{k-1} \frac{(m+1)[1+(m-2) \beta]-2(1-2 \beta-\gamma)}{(m+2)[1+(m-1) \beta]} .
\end{aligned}
$$

This completes the proof of Lemma 3.

Lemma 4. Let

$$
\eta>1, \quad 0 \leq \beta<\frac{2 \eta-2}{2 \eta^{2}+\eta-1} .
$$

Suppose also that $f \in \Sigma$ is given by (1) and

$$
\sum_{k=1}^{\infty}[k+\beta k(k-1)+\gamma]\left|a_{k}\right| \leq \gamma-1
$$

where (and throughout this paper unless otherwise mentioned) the parameter $\gamma$ is defined as

$$
\gamma:=\eta-\frac{1}{2} \beta\left(2 \eta^{2}+\eta+1\right)
$$

Then $f \in \mathscr{M}(\beta, \eta)$.

The proof of Lemma 4 is similar to that of Theorem 1 in Wang et al. [1] and so is omitted. 


\section{Main Results}

We begin by proving the following result which shows that $\mathscr{M}(\beta, \eta)$ is a subclass of $\mathscr{M}(\eta)$.

Theorem 5. Suppose that $\eta>1$ and $\beta \geq 0$. Then

$$
\mathscr{M}(\beta, \eta) \subset \mathscr{M}(\eta)
$$

Proof. Define

$$
\rho(z):=\frac{z f^{\prime}(z) / f(z)+\eta}{\eta-1} \quad(\eta>1 ; z \in \mathbb{U}) .
$$

Then $\rho$ is analytic in $\mathbb{U}$. It follows from (20) that

$$
-\frac{z f^{\prime \prime}(z)}{f^{\prime}(z)}=\eta+1-(\eta-1) \rho(z)+\frac{(\eta-1) z \rho^{\prime}(z)}{\eta-(\eta-1) \rho(z)} .
$$

Combining (20) and (21), we obtain that

$$
\begin{aligned}
\frac{z f^{\prime}(z)}{f(z)}\left(\beta \frac{z f^{\prime \prime}(z)}{f^{\prime}(z)}+1\right) \\
=\beta(\eta-1) z \rho^{\prime}(z)+\beta(\eta-1)^{2} \rho^{2}(z) \\
\quad-(\eta-1)(2 \beta \eta+\beta-1) \rho(z)+\eta(\beta \eta+\beta-1) \\
=\Phi\left(\rho(z), z \rho^{\prime}(z) ; z\right),
\end{aligned}
$$

where

$$
\begin{aligned}
\Phi(r, s ; t)= & \beta(\eta-1) s+\beta(\eta-1)^{2} r^{2} \\
& -(\eta-1)(2 \beta \eta+\beta-1) r+\eta(\beta \eta+\beta-1) .
\end{aligned}
$$

For all real $x$ and $y$ satisfying $y \leq-\left(1+x^{2}\right) / 2$, we have

$$
\begin{aligned}
\mathfrak{R}\{\phi & (i x, y ; z)\} \\
= & \beta(\eta-1) y-\beta(\eta-1)^{2} x^{2}+\eta(\beta \eta+\beta-1) \\
\leq & -\beta(\eta-1) \frac{1+x^{2}}{2}-\beta(\eta-1)^{2} x^{2} \\
& +\eta(\beta \eta+\beta-1) \leq \frac{1}{2} \beta\left(2 \eta^{2}+\eta+1\right)-\eta .
\end{aligned}
$$

If we set

$$
\Omega=\left\{\xi: \Re(\xi)>\frac{1}{2} \beta\left(2 \eta^{2}+\eta+1\right)-\eta\right\},
$$

then $\Phi(i x, y ; z) \notin \Omega$ for all real $x, y$ such that $y \leq$ $-\left(1+x^{2}\right) / 2$. Moreover, from definition (10), we know that $\Phi\left(\rho(z), z \rho^{\prime}(z) ; z\right) \in \Omega$. Using Lemma 2, we conclude that $\mathfrak{R}(\rho(z))>0$ for all $z \in \mathbb{U}$, which implies that $f \in \mathscr{M}(\eta)$. This completes the proof of Theorem 5 .

Now we consider the coefficient estimates for functions belonging to the class $\mathscr{M}(\beta, \eta)$.
Theorem 6. Suppose that

$$
\eta>1, \quad 0 \leq \beta<\frac{2 \eta-2}{2 \eta^{2}+\eta-1} .
$$

If $f \in \mathscr{M}(\beta, \eta)$, then

$$
\begin{gathered}
\left|a_{1}\right| \leq \frac{-1+2 \beta+\gamma}{1-\beta}, \\
\left|a_{k}\right| \leq \frac{-1+2 \beta+\gamma}{1-\beta} \\
\cdot \prod_{m=1}^{k-1} \frac{(m+1)[1+(m-2) \beta]-2(1-2 \beta-\gamma)}{(m+2)[1+(m-1) \beta]} \\
(k \in \mathbb{N} \backslash\{1\}) .
\end{gathered}
$$

Proof. Suppose that $f \in \mathscr{M}(\beta, \eta)$. Then there exists $\tau \in \mathscr{P}$ such that

$$
\frac{z f^{\prime}(z)}{f(z)}+\beta \frac{z^{2} f^{\prime \prime}(z)}{f(z)}+\gamma=(-1+2 \beta+\gamma) \tau(z) \quad\left(z \in \mathbb{U}^{*}\right) \text {. }
$$

It follows from (28) that

$$
z f^{\prime}(z)+\beta z^{2} f^{\prime \prime}(z)=[(-1+2 \beta+\gamma) \tau(z)-\gamma] f(z)
$$

Combining (1) and (29), we have

$$
\begin{aligned}
& \left(-\frac{1}{z}+\sum_{k=1}^{\infty} k a_{k} z^{k}\right)+\beta\left(\frac{2}{z}+\sum_{k=1}^{\infty} k(k-1) a_{k} z^{k}\right) \\
& =\left[(-1+2 \beta+\gamma)\left(1+\sum_{k=1}^{\infty} \tau_{k} z^{k}\right)-\gamma\right] \cdot\left(\frac{1}{z}+\sum_{k=1}^{\infty} a_{k} z^{k}\right) .
\end{aligned}
$$

Evaluating the coefficient of $z^{n}$ in both sides of (30) yields

$$
\begin{aligned}
& 2(1-\beta) a_{1}=(-1+2 \beta+\gamma) \tau_{2}, \\
(k+1)[1+(k-2) \beta] a_{k} & \\
= & (-1+2 \beta+\gamma)\left(\tau_{k+1}+\sum_{l=1}^{k-1} \tau_{k-l} a_{l}\right) .
\end{aligned}
$$

By observing the fact that $\left|\tau_{k}\right| \leq 2$ for $k \in \mathbb{N}$, we find from (31) and (32) that

$$
\begin{gathered}
\left|a_{1}\right| \leq \frac{-1+2 \beta+\gamma}{1-\beta} \\
\left|a_{k}\right| \leq \frac{2(-1+2 \beta+\gamma)}{(k+1)[1+(k-2) \beta]}\left[1+\sum_{m=1}^{k-1}\left|a_{m}\right|\right]
\end{gathered}
$$


Now we define the sequence $\left\{A_{k}\right\}_{k=1}^{\infty}$ as follows:

$$
\begin{gathered}
A_{1}=\frac{-1+2 \beta+\gamma}{1-\beta} \\
A_{k}=\frac{2(-1+2 \beta+\gamma)}{(k+1)[1+(k-2) \beta]}\left[1+\sum_{m=1}^{k-1} A_{m}\right] .
\end{gathered}
$$

In order to prove that

$$
\left|a_{k}\right| \leq A_{k} \quad(k \in \mathbb{N}),
$$

we use the principle of mathematical induction by noting that

$$
\left|a_{1}\right| \leq A_{1}=\frac{-1+2 \beta+\gamma}{1-\beta} .
$$

Therefore, we assume that

$$
\left|a_{m}\right| \leq A_{m} \quad(m=1,2, \ldots, k ; k \in \mathbb{N}) .
$$

Combining (32) and (33), we get

$$
\begin{aligned}
\left|a_{k+1}\right| & \leq \frac{2(-1+2 \beta+\gamma)}{(k+1)[1+(k-2) \beta]}\left[1+\sum_{m=1}^{k}\left|a_{m}\right|\right] \\
& \leq \frac{2(-1+2 \beta+\gamma)}{(k+1)[1+(k-2) \beta]}\left[1+\sum_{m=1}^{k} A_{m}\right]=A_{k+1} .
\end{aligned}
$$

Hence, by the principle of mathematical induction, we have

$$
\left|a_{k}\right| \leq A_{k} \quad(k \in \mathbb{N})
$$

as desired. By means of Lemma 2 and (33), we know that (12) holds. Combining (39) and (12), we readily get the coefficient estimates asserted by Theorem 6 .

Using Lemma 4, we introduce the $\delta$-neighborhood of a function $f \in \Sigma$ of the form (1) by means of the following definition:

$$
\begin{aligned}
\mathscr{N}_{\delta}(f):= & \left\{g \in \Sigma: g(z)=\frac{1}{z}+\sum_{k=1}^{\infty} b_{k} z^{k},\right. \\
& \left.\sum_{k=1}^{\infty} \frac{k+\beta k(k-1)+\gamma}{\gamma-1}\left|a_{k}-b_{k}\right| \leq \delta \quad(\delta \geq 0)\right\} .
\end{aligned}
$$

By making use of definition (40), we obtain the following result.

Theorem 7. If $f \in \sum$ satisfies the condition

$$
\frac{f(z)+\varepsilon z^{-1}}{1+\varepsilon} \in \mathscr{M}(\beta, \eta) \quad(\varepsilon \in \mathbb{C} ;|\varepsilon|<\delta ; \delta>0),
$$

then

$$
\mathcal{N}_{\delta}(f) \subset \mathscr{M}(\beta, \eta)
$$

Proof. It is easily seen from (10) that a function $g \in \mathscr{M}(\beta, \eta)$ if and only if

$$
\begin{array}{r}
\frac{z g^{\prime}(z)+\beta z^{2} g^{\prime \prime}(z)+g(z)}{z g^{\prime}(z)+\beta z^{2} g^{\prime \prime}(z)+(2 \gamma-1) g(z)} \neq \sigma \\
(z \in \mathbb{U} ; \sigma \in \mathbb{C} ;|\sigma|=1),
\end{array}
$$

which is equivalent to

$$
\frac{(g * \varrho)(z)}{z^{-1}} \neq 0 \quad(z \in \mathbb{U}),
$$

where

$$
\begin{gathered}
\varrho(z)=\frac{1}{z}+\sum_{k=1}^{\infty} c_{k} z^{k} \\
\left(c_{k}:=\frac{k+\beta k(k-1)+1-[k+\beta k(k-1)+(2 \gamma-1)] \sigma}{2 \beta+(-2+2 \beta+2 \gamma) \sigma}\right) .
\end{gathered}
$$

It follows from (45) that

$$
\begin{aligned}
\left|c_{k}\right| & \\
& =\left|\frac{k+\beta k(k-1)+1-[k+\beta k(k-1)+(2 \gamma-1)] \sigma}{2 \beta+(-2+2 \beta+2 \gamma) \sigma}\right| \\
& \leq \frac{k+\beta k(k-1)+1+[k+\beta k(k-1)+(2 \gamma-1)]|\sigma|}{(-2+2 \beta+2 \gamma)|\sigma|-2 \beta} \\
& =\frac{k+\beta k(k-1)+\gamma}{\gamma-1} \quad(|\sigma|=1) .
\end{aligned}
$$

Furthermore, under the hypotheses of Theorem 7, (44) yields the following inequality:

$$
\left|\frac{(f * \varrho)(z)}{z^{-1}}\right| \geq \delta \quad(z \in \mathbb{U} ; \delta>0) .
$$

Suppose that

$$
\chi(z)=\frac{1}{z}+\sum_{k=1}^{\infty} d_{k} z^{k} \in \mathcal{N}_{\delta}(f) .
$$

It follows from (40) that

$$
\begin{aligned}
\left|\frac{((f-\chi) * \varrho)(z)}{z^{-1}}\right| & =\left|\sum_{k=1}^{\infty}\left(a_{k}-d_{k}\right) c_{k} z^{k+1}\right| \\
& \leq|z| \sum_{k=1}^{\infty} \frac{k+\beta k(k-1)+\gamma}{\gamma-1}\left|a_{k}-d_{k}\right|<\delta .
\end{aligned}
$$

Combining (47) and (49), we have

$$
\begin{aligned}
\left|\frac{(\chi * \varrho)(z)}{z^{-1}}\right| & =\left|\frac{([f+(\chi-f)] * \varrho)(z)}{z^{-1}}\right| \\
& \geq\left|\frac{(f * \varrho)(z)}{z^{-1}}\right|-\left|\frac{((\chi-f) * \varrho)(z)}{z^{-1}}\right|>0,
\end{aligned}
$$


which implies that

$$
\frac{(\chi * \varrho)(z)}{z^{-1}} \neq 0 \quad(z \in \mathbb{U}) .
$$

Thus, we have

$$
\chi(z) \in \mathcal{N}_{\delta}(f) \subset \mathscr{M}(\beta, \eta) .
$$

This completes the proof of Theorem 7.

Finally, we derive the partial sums of functions in the class $\mathscr{M}(\beta, \eta)$.

Theorem 8. Let $f \in \Sigma$ be given by (1) and define the partial sums $f_{n}(z)$ of $f$ by

$$
f_{n}(z)=\frac{1}{z}+\sum_{k=1}^{n} a_{k} z^{k} \quad(n \in \mathbb{N}) .
$$

Suppose also that

$$
\sum_{k=1}^{\infty} \frac{k+\beta k(k-1)+\gamma}{\gamma-1}\left|a_{k}\right| \leq 1 .
$$

Then

(1) $f \in \mathscr{M}(\beta, \eta)$;

(2)

$\Re\left(\frac{f(z)}{f_{n}(z)}\right) \geq \frac{n+\beta n(n+1)+2}{n+\beta n(n+1)+1+\gamma} \quad(n \in \mathbb{N} ; z \in \mathbb{U})$,

$$
\Re\left(\frac{f_{n}(z)}{f(z)}\right) \geq \frac{n+\beta n(n+1)+1+\gamma}{n+\beta n(n+1)+2 \gamma} \quad(n \in \mathbb{N} ; z \in \mathbb{U}) .
$$

Each of the bounds in (55) and (56) is the best possible for each $n \in \mathbb{N}$.

Proof. (1) It is easy to see that the result follows directly from Lemma 4.

(2) Note that

$$
\frac{n+1+\beta n(n+1)+\gamma}{\gamma-1}>\frac{n+\beta n(n-1)+\gamma}{\gamma-1}>1 \quad(n \in \mathbb{N}) .
$$

Thus, we have

$$
\begin{gathered}
\sum_{k=1}^{n}\left|a_{k}\right|+\frac{n+\beta n(n+1)+1+\gamma}{\gamma-1} \sum_{k=n+1}^{\infty}\left|a_{k}\right| \\
\leq \sum_{k=1}^{\infty} \frac{k+\beta k(k-1)+\gamma}{\gamma-1}\left|a_{k}\right| \leq 1 .
\end{gathered}
$$

By setting

$$
\begin{aligned}
h_{1}(z)= & \frac{n+\beta n(n+1)+1+\gamma}{\gamma-1} \\
& \cdot\left(\frac{f(z)}{f_{n}(z)}-\frac{n+\beta n(n+1)+2}{n+\beta n(n+1)+1+\gamma}\right) \\
= & 1+\frac{((n+\beta n(n+1)+1+\gamma) /(\gamma-1)) \sum_{k=n+1}^{\infty} a_{k} z^{k+1}}{1+\sum_{k=1}^{n} a_{k} z^{k+1}},
\end{aligned}
$$

we find from (58) and (59) that

$\left|\frac{h_{1}(z)-1}{h_{1}(z)+1}\right|$

$$
\begin{aligned}
& \leq \frac{((n+\beta n(n+1)+1+\gamma) /(\gamma-1)) \sum_{k=n+1}^{\infty}\left|a_{k}\right|}{2-2 \sum_{k=1}^{n}\left|a_{k}\right|-((n+\beta n(n+1)+1+\gamma) /(\gamma-1)) \sum_{k=n+1}^{\infty}\left|a_{k}\right|} \\
& \leq 1 \quad(z \in \mathbb{U}),
\end{aligned}
$$

which implies inequality (55).

$$
\text { If we put }
$$

$$
f(z)=\frac{1}{z}-\frac{\gamma-1}{n+\beta n(n+1)+1+\gamma} z^{n+1},
$$

then

$$
\begin{gathered}
\frac{f(z)}{f_{n}(z)}=1-\frac{\gamma-1}{n+\beta n(n+1)+1+\gamma} z^{n+2} \\
\longrightarrow \frac{n+\beta n(n+1)+2}{n+\beta n(n+1)+1+\gamma} \\
\left(z \longrightarrow 1^{-}\right),
\end{gathered}
$$

which shows that the bound in (55) is the best possible for each $n \in \mathbb{N}$.

Now, we set

$$
\begin{aligned}
h_{2}(z)= & \frac{n+\beta n(n+1)+2 \gamma}{\gamma-1} \\
& \cdot\left(\frac{f_{n}(z)}{f(z)}-\frac{n+\beta n(n+1)+1+\gamma}{n+\beta n(n+1)+2 \gamma}\right) \\
= & 1-\frac{((n+\beta n(n+1)+2 \gamma) /(\gamma-1)) \sum_{k=n+1}^{\infty} a_{k} z^{k+1}}{1+\sum_{k=1}^{\infty} a_{k} z^{k+1}} .
\end{aligned}
$$

In view of (58) and (63), we conclude that

$$
\begin{aligned}
& \left|\frac{h_{2}(z)-1}{h_{2}(z)+1}\right| \\
& \leq \frac{((n+\beta n(n+1)+2 \gamma) /(\gamma-1)) \sum_{k=n+1}^{\infty}\left|a_{k}\right|}{2-2 \sum_{k=1}^{n}\left|a_{k}\right|-((n+\beta n(n+1)+2) /(\gamma-1)) \sum_{k=n+1}^{\infty}\left|a_{k}\right|} \\
& \leq 1 \quad(z \in \mathbb{U}),
\end{aligned}
$$


which leads to inequality (56) asserted in Theorem 8 . The bound in (56) is sharp with the extremal function $f$ given by (61). We thus complete the proof of Theorem 8 .

In what follows, we turn to quotients involving derivatives. The proof of Theorem 9 is similar to that of Theorem 8 and so the details may be omitted.

Theorem 9. Let $f \in \Sigma$ be given by (1) and define the partial sums $f_{n}(z)$ of $f$ by (53). If the condition (54) holds, then

$$
\begin{aligned}
& \Re\left(\frac{f^{\prime}(z)}{f_{n}^{\prime}(z)}\right) \geq \frac{\beta n(n+1)-n \gamma}{n+\beta n(n+1)+1+\gamma} \quad(n \in \mathbb{N} ; z \in \mathbb{U}), \\
& \Re\left(\frac{f_{n}^{\prime}(z)}{f^{\prime}(z)}\right) \geq \frac{n+\beta n(n+1)+1+\gamma}{\beta n(n+1)+(n+2) \gamma} \quad(n \in \mathbb{N} ; z \in \mathbb{U}) .
\end{aligned}
$$

The bounds in (65) are sharp with the extremal function given by (61).

\section{Conflict of Interests}

The authors declare that they have no competing interests.

\section{Authors' Contribution}

The authors jointly worked on the results and they read and approved the final paper.

\section{Acknowledgments}

The present investigation was supported by the National Natural Science Foundation under Grant nos. 11301008 and 11426035 and the Foundation for Excellent Youth Teachers of Colleges and Universities of Henan Province under Grant no. 2013GGJS-146.

\section{References}

[1] Z.-G. Wang, Y. Sun, and Z.-H. Zhang, "Certain classes of meromorphic multivalent functions," Computers \& Mathematics with Applications, vol. 58, no. 7, pp. 1408-1417, 2009.

[2] Z. Nehari and E. Netanyahu, "On the coefficients of meromorphic schlicht functions," Proceedings of the American Mathematical Society, vol. 8, pp. 15-23, 1957.

[3] R. M. Ali and V. Ravichandran, "Classes of meromorphic $\alpha$ convex functions," Taiwanese Journal of Mathematics, vol. 14, no. 4, pp. 1479-1490, 2010.

[4] M. K. Aouf and A. O. Mostafa, "On partial sums of certain meromophic p-valent functions," Mathematical and Computer Modelling, vol. 50, no. 9-10, pp. 1325-1331, 2009.

[5] A. Cãtaş, "Some simple criteria of starlikeness for meromorphic functions," Filomat, vol. 22, no. 2, pp. 109-113, 2008.

[6] J. Dziok, "Classes of meromorphic functions associated with conic regions," Acta Mathematica Scientia, vol. 32, no. 2, pp. 765-774, 2012.

[7] J. Dziok, "Classes of multivalent analytic and meromorphic functions with two fixed points," Fixed Point Theory and Applications, vol. 2013, article 86, 2013.
[8] J.-L. Liu and H. M. Srivastava, "Classes of meromorphically multivalent functions associated with the generalized hypergeometric function," Mathematical and Computer Modelling, vol. 39, no. 1, pp. 21-34, 2004.

[9] V. Ravichandran, C. Selvaraj, and R. Rajalaksmi, "Sufficient conditions for starlike functions of order $\alpha$, Journal of Inequalities in Pure and Applied Mathematics, vol. 3, no. 5, article 81, 2002.

[10] M.-S. Liu, Y.-C. Zhu, and H. M. Srivastava, "Properties and characteristics of certain subclasses of starlike functions of order $\beta$," Mathematical and Computer Modelling, vol. 48, no. 34, pp. 402-419, 2008.

[11] S. Singh and S. Gupta, "Starlikeness of analytic maps satisfying a differential inequality," General Mathematics, vol. 18, no. 3, pp. 51-58, 2010.

[12] Z.-G. Wang, X.-S. Yuan, and L. Shi, "Neighborhoods and partial sums of certain subclass of starlike functions," Journal of Inequalities and Applications, vol. 2013, article 163, 2013.

[13] Z.-G. Wang, H. M. Srivastava, and S.-M. Yuan, "Some basic properties of certain subclasses of meromorphically starlike functions," Journal of Inequalities and Applications, vol. 2014, article 29, 2014.

[14] S. S. Miller and P. T. Mocanu, "Differential subordinations and inequalities in the complex plane," Journal of Differential Equations, vol. 67, no. 2, pp. 199-211, 1987. 


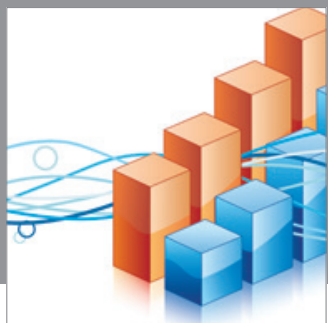

Advances in

Operations Research

mansans

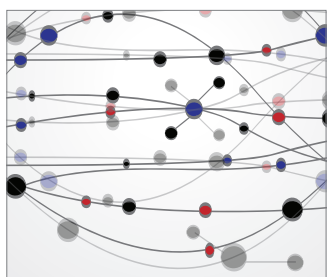

The Scientific World Journal
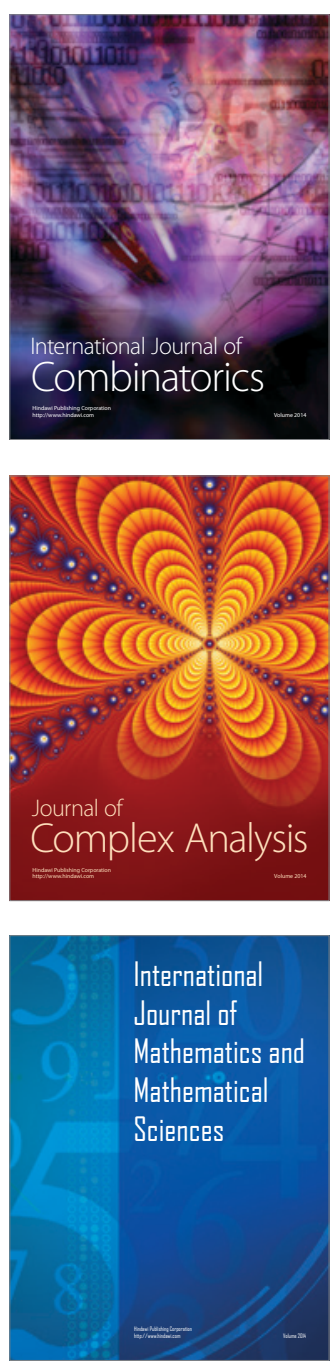
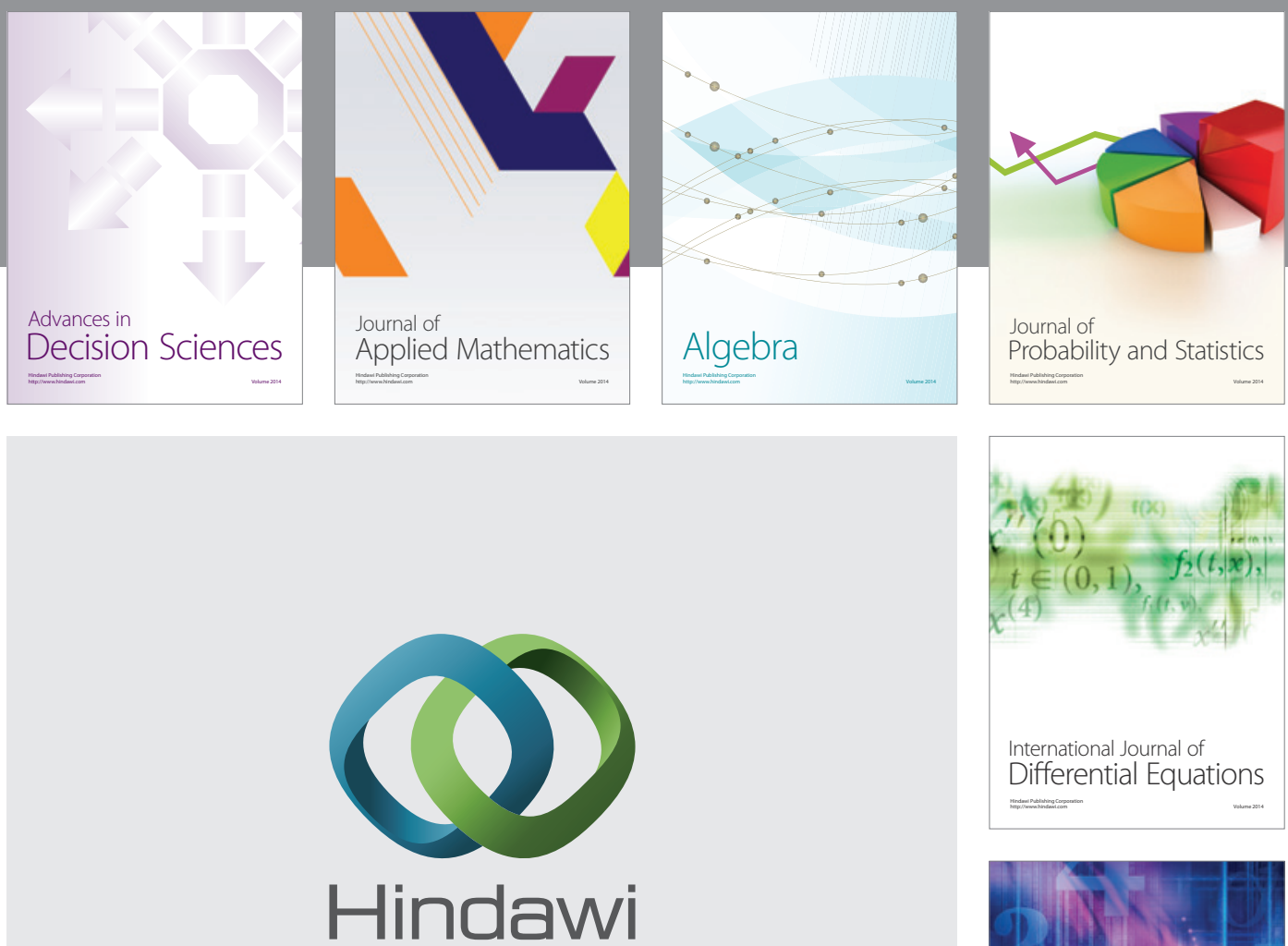

Submit your manuscripts at http://www.hindawi.com
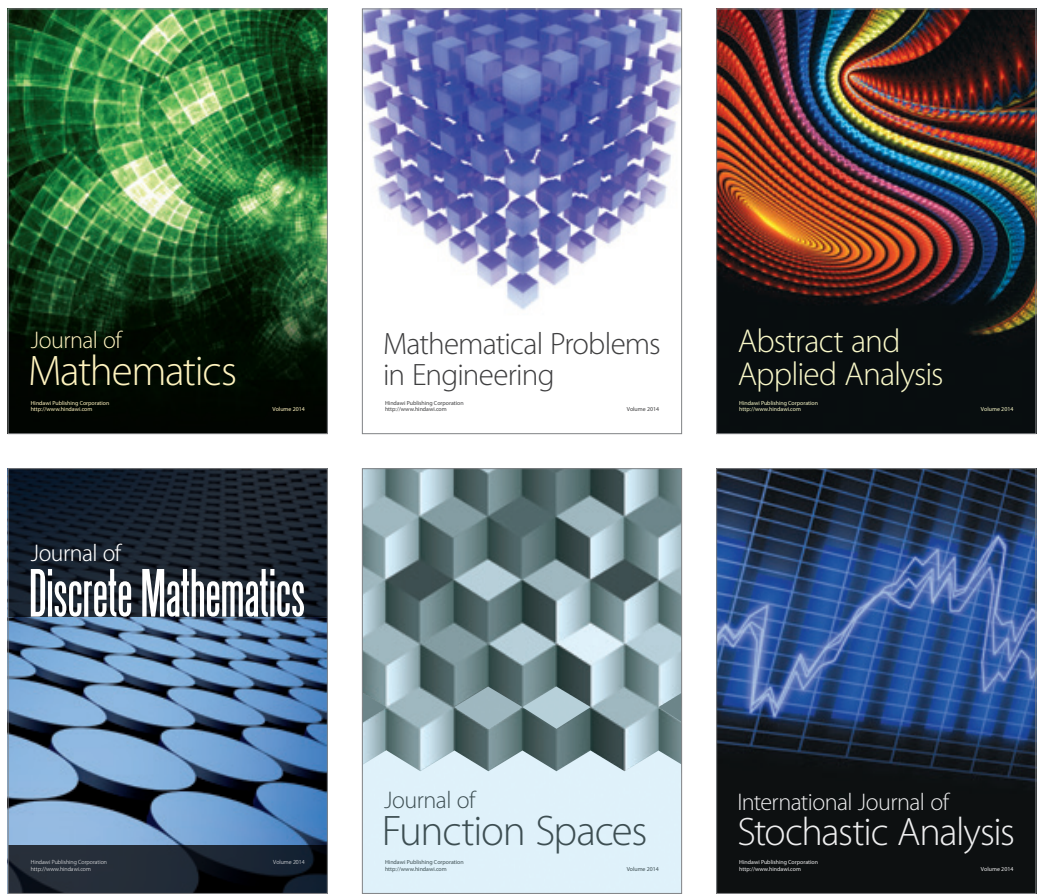

Journal of

Function Spaces

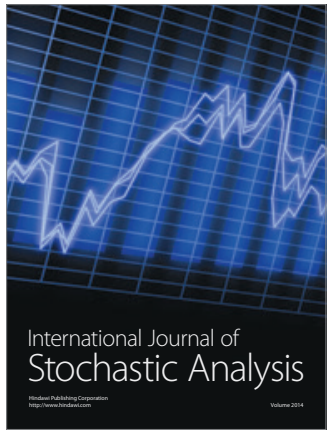

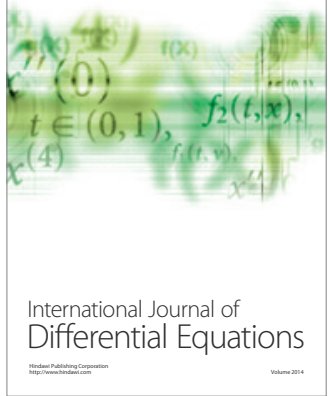
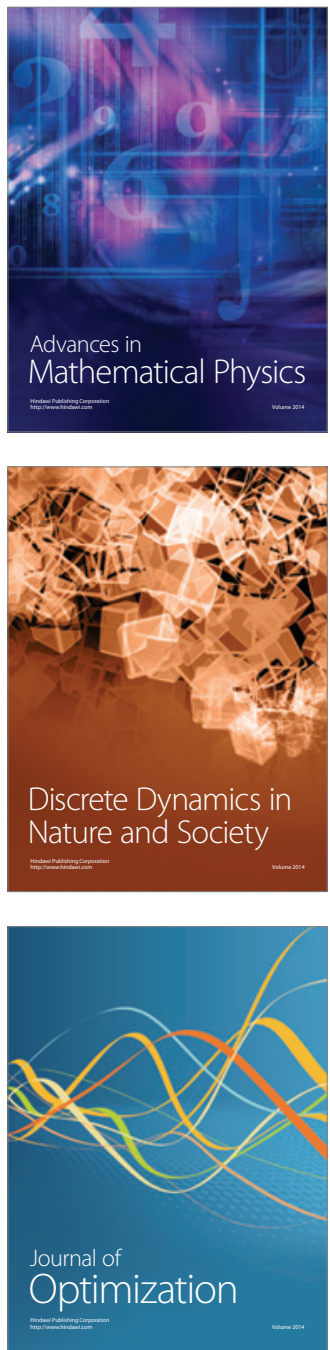